\title{
Breast cancer incidence and overdiagnosis in Catalonia (Spain)
}

Montserrat Martinez-Alonso ${ }^{1,2 \dagger}$, Ester Vilaprinyo ${ }^{3 \dagger}$, Rafael Marcos-Gragera ${ }^{4,5}$, Montserrat Rue ${ }^{1,2^{*}}$

\begin{abstract}
Introduction: Early detection of breast cancer (BC) with mammography may cause overdiagnosis and overtreatment, detecting tumors which would remain undiagnosed during a lifetime. The aims of this study were: first, to model invasive BC incidence trends in Catalonia (Spain) taking into account reproductive and screening data; and second, to quantify the extent of BC overdiagnosis.
\end{abstract}

Methods: We modeled the incidence of invasive BC using a Poisson regression model. Explanatory variables were: age at diagnosis and cohort characteristics (completed fertility rate, percentage of women that use mammography at age 50, and year of birth). This model also was used to estimate the background incidence in the absence of screening. We used a probabilistic model to estimate the expected BC incidence if women in the population used mammography as reported in health surveys. The difference between the observed and expected cumulative incidences provided an estimate of overdiagnosis.

Results: Incidence of invasive BC increased, especially in cohorts born from 1940 to 1955. The biggest increase was observed in these cohorts between the ages of 50 to 65 years, where the final $B C$ incidence rates more than doubled the initial ones. Dissemination of mammography was significantly associated with BC incidence and overdiagnosis. Our estimates of overdiagnosis ranged from 0.4\% to 46.6\%, for women born around 1935 and 1950, respectively.

Conclusions: Our results support the existence of overdiagnosis in Catalonia attributed to mammography usage, and the limited malignant potential of some tumors may play an important role. Women should be better informed about this risk. Research should be oriented towards personalized screening and risk assessment tools.

\section{Introduction}

Breast cancer $(\mathrm{BC})$ incidence rates in women have been increasing steadily in the $1980 \mathrm{~s}$ and $1990 \mathrm{~s}$ in many countries. Time trends in the incidence of breast cancer have been influenced by different factors: changes in reproductive patterns, the introduction of screening mammography, obesity trends, hormone replacement therapy (HRT), oral contraceptive use and better health awareness [1-10]. In Europe, BC incidence increased in all countries, with or without national screening programs [6]. In Spain, where BC incidence is lower than the European average, incidence rates have increased since 1973, with a more marked rise during the $1990 \mathrm{~s}$

\footnotetext{
* Correspondence: montse.rue@cmb.udl.cat

+ Contributed equally

'Basic Medical Sciences Department, Biomedical Research Institut of Lleida (IRBLLEIDA), Alcalde Rovira Roure 80, Lleida, 25198, Catalonia, Spain

Full list of author information is available at the end of the article
}

[11] in parallel with the dissemination of mammography, both opportunistic and publicly organized screening programs. In Catalonia (Spain), the annual percentage change between 1980 to 1984 and 1995 to 1999 was $2.2 \%$ [12] and new cases of female BC were estimated at 4,700 in the year 2008 . This quantity represents $30 \%$ of all cancer diagnoses in women.

Screening increases incidence rates in three ways [13]. First, there is an immediate rise in incidence, due to the early diagnosis of prevalent asymptomatic cancers $[14,15]$. Second, age at diagnosis decreases as a result of the lead time introduced by screening (estimated as two to four years) [16]. Third, screening may cause overdiagnosis when it detects tumors which would never have been diagnosed during a lifetime without screening because of the lack of progressive potential or death from other causes [17-21].

\section{Biomed Central}


Estimates of overdetection of invasive cancer may be affected by important biases. Biesheuvel et al. mentioned 1) differences in the underlying breast cancer risk between screened and unscreened populations, 2) contamination of intervention and control groups, 3) screening in the control group after the intervention period ends, and 4) inadequate allowance for lead-time [22]. It seems that the older trial data tend to provide lower estimates of overdiagnosis, whereas the more recent observational data tend toward higher estimates. Since no decline in interval cancer rates has been observed [23] part of the overdiagnosis increase may be related to an increase in the sensitivity of mammography (both for cancers that will progress and for tumors of limited malignant potential).

Mathematical modeling may overcome some of the previous mentioned biases. In the US, Holford estimated the contribution of screening to the upward trend in $\mathrm{BC}$ incidence using log-linear models for age, period and birth cohort (APC) [24]. Holford's models also provided background estimates of trends that might have been expected as a result of the continuation of historical increases in the rates. The Cancer Intervention and Surveillance Modeling Network (CISNET) used the background estimates as inputs for modeling the impact of screening and adjuvant treatments on $\mathrm{BC}$ mortality trends $[25,26]$.

The interest in assessing the impact and cost-effectiveness of breast cancer early detection programs in Catalonia (Spain) led us to work with mathematical models developed by Lee and Zelen within the CISNET [27,28]. The aims of this study are 1) to use reproductive and screening data to model invasive $\mathrm{BC}$ incidence trends and to obtain background estimates of invasive $\mathrm{BC}$ incidence and 2) to use the Lee and Zelen mathematical models to quantify the extent of overdetection of invasive $\mathrm{BC}$ related to screening.

\section{Materials and methods Setting}

In Spain there is a National Health System (NHS), financed primarily by taxes, which provides universal and free health coverage, including early detection of breast cancer. Catalonia is an autonomous region of Spain which has approximately one sixth of the Spanish population. The Catalan Breast Cancer Screening Program (BCSP) started gradually, at the beginning of the $1990 \mathrm{~s}$, providing biennial mammography screening tests to women 50 to 64 years old. Since the year 2000, women older than 64 are kept in the program until the age of 69 . Before the start of and in addition to the BCSP, there has also been a certain degree of opportunistic breast cancer screening done in the public and private health care sectors. In the 1994 Catalan Health
Survey, when most of the screening in Catalonia was opportunistic, rates of screening mammography were $43 \%$ in women aged 40 to 49 years and $27 \%$ in women aged 50 to 64 years [29]. In 2004, $61.2 \%$ of the invited women participated in the BCSP and $75.7 \%$ either participated in the BCSP or reported that they had received recent mammograms (non-published BCSP data).

\section{Data}

To model invasive breast cancer incidence rates in Catalonia, age and period specific incidence data were obtained from the Girona and Tarragona populationbased cancer registries in Catalonia (both provinces representing $18.5 \%$ of the total Catalan population and covering either urban and rural areas). The Girona and Tarragona provinces have around 750,000 and 800,000 inhabitants, respectively. Data from Girona was provided directly by the Girona Cancer Registry and data from Tarragona was downloaded from the International Agency for Research on Cancer registries (IARC) [11]. Incidence data were available for calendar years 1980 to 1989 and 1991 to 2004 for Girona and 1983 to 1997 for Tarragona. Given that the breast cancer incidence rates in the Girona and Tarragona registries are similar [30], both data sources were combined. Numerators for the incidence rates were calculated adding the number of incident cases from both registries by age and calendar year. Denominators were calculated adding the number of women at mid-calendar year, in the Girona and Tarragona provinces, and were obtained from official census population data [31]. We have assumed that the estimated incidence rates were representative of the breast cancer incidence in Catalonia. Ductal carcinoma in situ (DCIS) has not been included in the analysis.

The research protocol was approved by the institutional review board and ethics committee of the Hospital Universitari de Bellvitge (Barcelona) which waived the need for informed consent.

\section{Breast cancer incidence models}

We modeled the observed incidence of invasive $\mathrm{BC}$ for Catalan women aged 25 to 84 during the time period 1980 to 2004 using an age-cohort model that incorporated cohort characteristics like intensity of mammography utilization and fecundity rate. We did not introduce a period effect because screening mammography was disseminated gradually in our country. We used this model to estimate the background incidence of $\mathrm{BC}$ under the assumption of no screening. Then, using a probabilistic model that takes into account background incidence, competing risks, distribution of sojourn time in preclinical state, sensitivity of mammography and the dissemination of screening in Catalonia, we estimated the increased age-specific incidence due to lead time 
(expected incidence). Finally, we estimated overdiagnosis comparing the observed and expected cumulative incidences, by cohort of birth. In the following sections there are the models' details.

\section{Observed incidence model}

Observed incidence rates were fitted using an agecohort model where the cohort effects were split into three components. The first component refers to the fecundity of the cohort and was measured using the cohorts' completed fertility rate (CFR). CFR is the average number of births per woman up to the end of the childbearing years [32]. There is evidence that high parity is protective of breast cancer independent of ages at first and last full term pregnancies [33]. The second component refers to the intensity that mammography was used for each specific cohort and was measured as the proportion of women who were having periodic mammograms for early detection at age 50 (PM50). This value was obtained from a previous work of modeling mammography dissemination in Catalonia (see the details in Appendix A in Additional file 1) [34]. Finally, the third component refers to the remaining cohort characteristics once fecundity and mammography use have been taken into account and for which we do not have data over time, like use of HRT, use of oral contraceptives, obesity, diet or health awareness. The third component has been included in the model as the year of birth of the cohorts.

Our BC incidence model uses age, CFR, PM50 and year of birth to estimate the number of $\mathrm{BC}$ incident cases by age and cohort of birth. It assumes a Poisson distribution of the incident cases and takes into account the exposed population. We used fractional polynomials to describe the age and cohort effects in order to increase the exibility of conventional polynomial models and avoid undesirable artifacts of highorder curves (see Appendix B in Additional file 1 and reference [35] for more detail). Since mammography use (PM50) and CFR had opposite trends in most of the studied periods, and PM50 was strongly associated with $\mathrm{BC}$ incidence, we decided to include CFR in the model as an offset with the coefficient -0.15 . This value was obtained from the literature and indicates a relative risk of $\mathrm{BC}$ equal to 0.85 for each child born $[1,2,36]$.

Goodness of fit was assessed using the deviance and the likelihood ratio test with respect to the saturated model. Overdispersion of the Poisson model was assessed. Plots of residuals versus fitted values and predictors were assessed to check for lack of fit related to the scale of predictors (data not shown). Confidence intervals of the predicted values were obtained using the delta method and bootstrap.

\section{Background incidence model}

$\mathrm{BC}$ incidence in absence of screening, by cohort of birth, was derived from the $\mathrm{BC}$ observed incidence model by considering that the proportion of women having periodic mammograms at age 50 (PM50) was zero. Confidence intervals for background incidence were obtained using the delta method and bootstrap.

\section{Expected incidence and overdiagnosis estimation}

Using the probabilistic model developed by Lee and Zelen (LZ) for the CISNET [28] we estimated expected $\mathrm{BC}$ incidence if women in the population had used mammography as they reported in health surveys, and overdiagnosis was zero. This estimate takes into account the lead time that results when breast cancer is diagnosed earlier. Then the difference between the observed and expected incidence provided an estimate of overdiagnosis. The steps were the following (Figure 1):

1. We considered all the scenarios that assumed a) $100 \%$ of women starting screening mammography at age $z$ with $40 \leq z \leq w_{\max }$, where $w_{\max }$ is the highest age attained by each cohort and b) the periodicity of the exams was annual or biennial. For instance, for cohort $1950, w_{\max }$ was 54 , then $z$ took 15 different values. Therefore, for this cohort 30 different scenarios were computed (15 with annual screening and 15 with biennial screening).

2. We used the LZ model to estimate the number of breast cancer incident cases by age and cohort of birth in each of the scenarios mentioned in step 1, assuming that each cohort had 100,000 women at birth. See the equations in Appendices $\mathrm{C}$ and $\mathrm{D}$ in Additional file 1 and [28,37-40] for more detail.

3. We considered the dissemination of mammography use in Catalonia by age and birth cohort in order to obtain the scenario that best represents the real pattern of mammography use for each cohort. Weighting the estimates obtained for each scenario in step two by the pattern of mammography use, we obtained the estimated number of incident cases by age and birth cohort (see Appendix B in Additional file 1).

4. For each cohort we obtained the expected cumulative incidence (per 100,000 women at birth) adding the incident cases obtained in step three. We represent this estimate by $C I_{e}$.

5 . We estimated the observed cumulative incidence by 100,000 women at birth $\left(C I_{o}\right)$ multiplying the observed age-specific incidence rates by the probability of being alive at each age, and adding up all these values.

Finally the estimates of overdiagnosis by cohort of birth were obtained using the formula:

$$
100 \times \frac{\mathrm{Cl}_{O}-\mathrm{Cl} e}{\mathrm{Cl} l_{e}}
$$


Appendix E in Additional file 1 shows the calculation of overdiagnosis for the cohort born in 1950 as an illustrative example.

The steps taken to obtain confidence intervals of the overdiagnosis estimates are described in Appendix E.2 in Additional file 1.

\section{Sensitivity of the overdiagnosis estimates to changes in relevant parameters}

We obtained new estimates of overdiagnosis by modifying the following parameters:

1. Mean sojourn time in pre-clinical state $(\alpha)$. In the LZ model $\alpha$ takes values in the range of two to four years, depending on age. We estimated overdiagnosis when $\alpha=1$ and when $\alpha=5$ for all ages. These scenarios would represent tumors growing faster or slower than in the LZ model, respectively.

2. Mammography sensitivity $(\beta)$. In the LZ model $\beta$ varies from 0.35 to 0.8 , depending on age of the woman and year when mammography was performed. We estimated the overdiagnosis assuming $\beta=0.9$ for all ages.

3. Repeat mammography behavior. Since the distributions of periodicity of mammograms in Catalonia were quite stable along different ages and calendar years, we obtained new estimates of overdiagnosis in the most extreme situations:

a. 1994 Health Survey for the age-group 40 to 49 years (annual $=0.76$, biennial $=0.21$, irregular $=0.04$ ).

b. 2006 Health Survey for ages from 60 to 69 years (annual $=0.52$, biennial $=0.35$, irregular $=0.13$ ).

\section{Software}

The Stata SE/10 statistical package was used to fit the Poisson model for $\mathrm{BC}$ incidence, to bootstrap the residuals and to obtain confidence intervals [41]. The Grid Mathematica v6 program was used to apply the stochastic LZ model, to estimate the number of BC incident cases under different screening scenarios, and to estimate overdiagnosis [42].

\section{Results}

Figure 2 shows the trend over time of the completed fertility rate (CFR) (2a) and of the proportion of women receiving periodic mammograms at age 50 (PM50) (2b), two of the cohort variables used to model $\mathrm{BC}$ incidence. 

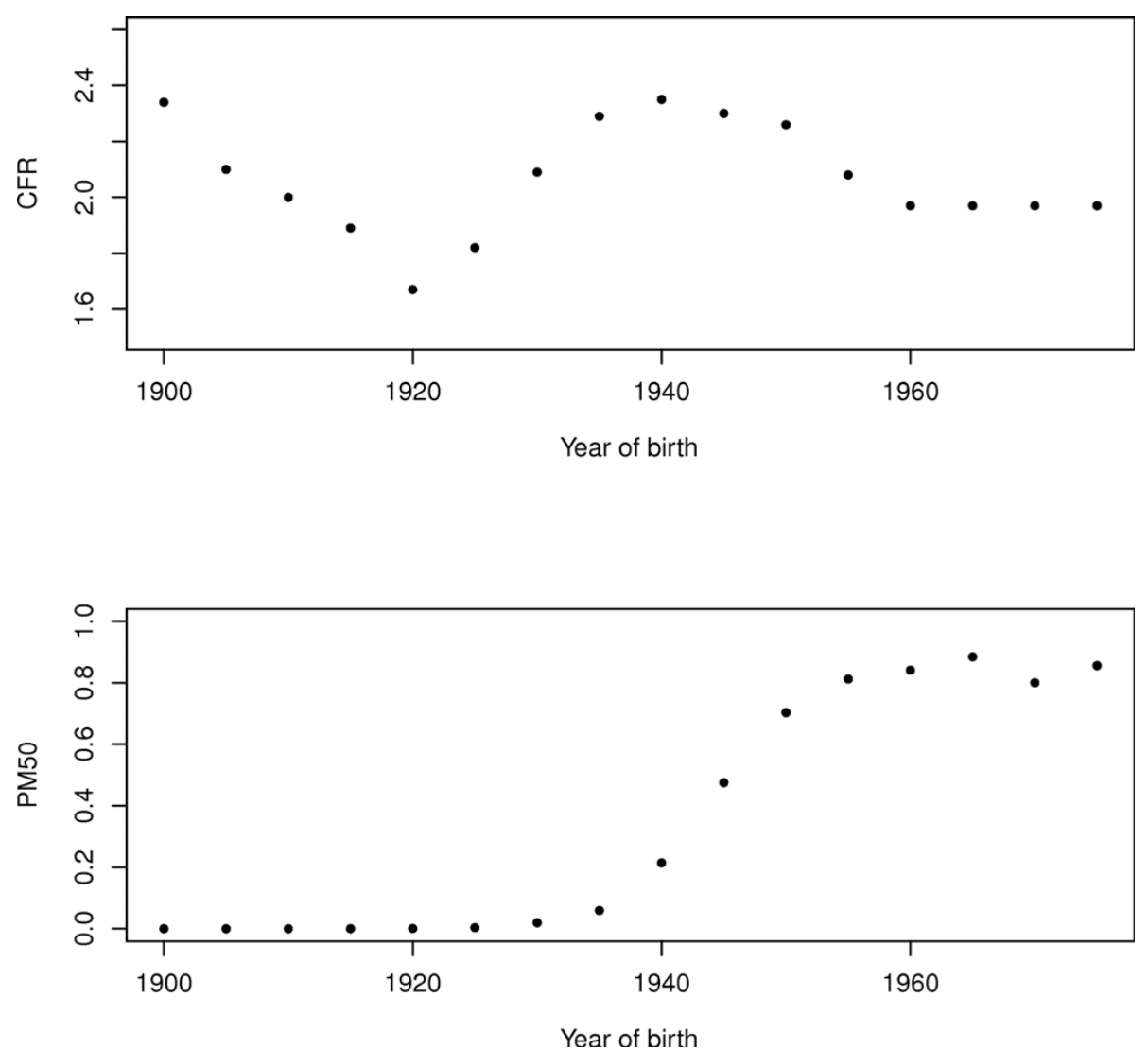

Figure 2 Completed fertility rate (CFR) and proportion having periodic mammograms at age 50 (PM50).

Figure 2a shows that CFR decreased for women born between 1900 and 1920 and then increased from 1920 to 1940 , reaching a maximum of 2.35 children for women born in 1940. After, the CFR decreased again. Figure $2 \mathrm{~b}$ shows a big increase in mammography usage from 1935 to 1955. For a woman born after 1955 the values of PM50 stabilized at approximately 0.8 . It is important to note that for women born between 1940 and 1955 we observed both a decrease of CFR and an increase of PM50.

Table 1 shows the equation, the estimated coefficients and standard errors of the BC incidence model. Fractional polynomials selected powers two and three for age, lineal for year of birth and power 0.5 for PM50. Independent variables were centered with respect their means. The model coefficients indicate that incidence increased with age, year of birth and exposure to periodic mammography. The square root effect of PM50 on $\mathrm{BC}$ incidence indicates that the impact of mammography use in BC incidence attenuated as PM50 increased. The effect of fecundity on $\mathrm{BC}$ incidence was considered inverse and constant overtime.

The observed $\mathrm{BC}$ incidence rates and the estimation provided by the model can be observed in Figure 3 grouped by year of birth. There was agreement between observed and predicted values. Data shows an increase in BC incidence, especially in cohorts born from 1940 to 1955. The biggest increase of $\mathrm{BC}$ incidence was observed for ages 50 to 65 years, where the lowest BC incidence rate was less than half of the highest. The slopes of the estimated rates for the oldest cohorts were nearly parallel but we observed increasingly steeper slopes for the 1935 and younger cohorts.

Table 1 Breast cancer incidence model; Catalonia 1980 to 2004

\begin{tabular}{lccc}
\hline & Coef. & Std. error & $P$-value \\
\hline Age $_{1}$ & -38.8418 & 1.0854 & $<0.001$ \\
Age $_{2}$ & 0.0005 & 0.0002 & 0.002 \\
$\mathrm{PM50}_{1}$ & 0.6250 & 0.9878 & $<0.001$ \\
$\mathrm{YB}_{1}$ & 0.0111 & 0.0026 & $<0.001$ \\
Constant & -6.0626 & 0.0156 & $<0.001$ \\
\hline
\end{tabular}

The dependent variable is the number of observed incidence cases. The model has an offset, which is a term with coefficient constrained to 1 . offset $=$ $\log \left(\right.$ exposed) 0.15 CFR, $\mathrm{Age}_{1}=(\mathrm{age} / 10)^{-2}-0.0331, \mathrm{Age}_{2}=(\mathrm{age} / 10)^{3}-166.375$, $\mathrm{PM} 50_{1}=\mathrm{PM} 50^{0.5}-0.4342, \mathrm{YB}_{1}=$ year-of-birth-1937.5. CFR: completed fertility rate, PM50: proportion of women who were having periodic mammograms for early detection at age 50 . Deviance $=482.53$. The expected number of incident cases, $E(I)$, can be obtained using the equation: $E(I)=\exp (-6.0626$ $38.8418 \mathrm{Age}_{1}+0.0005 \mathrm{Age}_{2}+0.6250 \mathrm{PM} 50_{1}+0.0111 \mathrm{YB}_{1}+$ offset $)$. 


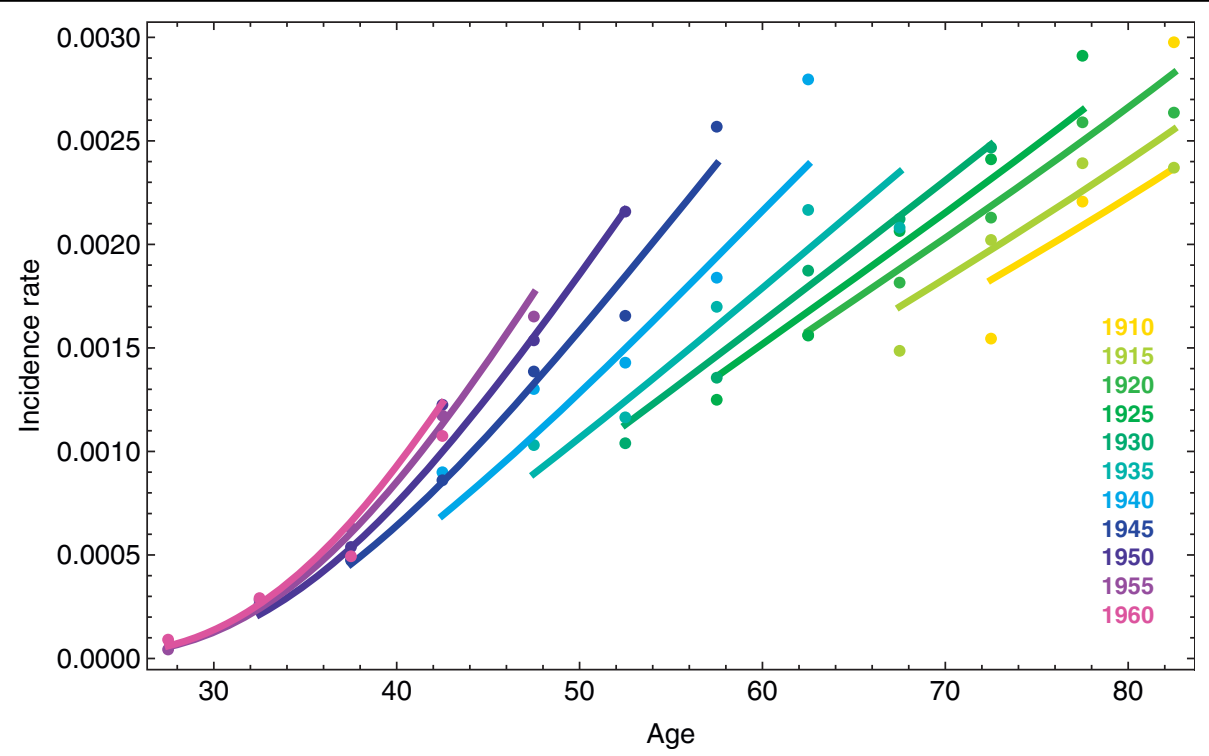

Figure 3 Observed breast cancer (BC) incidence rates per 100,000 women (points) and the fitted model (lines). Each color represents a cohort of birth.

Figure 4 shows the observed (dots), estimated (blue dashed line) and background incidence rates for cohorts 1935, 1940, 1945 and 1950 (purple line). Confidence intervals correspond to the bootstrap method and show narrow estimations (intervals obtained by the delta method were slightly narrower). Differences between background and screening scenarios were insignificant for woman born in 1935 or before (data not shown) and increasingly higher for woman born later. This provided the first clue to the magnitude of overdiagnosis due to screening.

Figure 5 presents the number of incident $\mathrm{BC}$ cases that would be obtained from a cohort of 100,000 women at birth in three different situations. The dashed line indicates the number of cases under the background incidence scenario and represents a situation of no mammography use. The solid line indicates the number of cases that would be diagnosed if mammography had been used as reported in the Health Surveys. These estimates were obtained by applying the LZ model to the background incidence. The increase in incidence with respect to the background is due to early diagnosis. Dots represent the number of observed cases for 100,000 women at birth and have been obtained from the observed incidence rates and probabilities of survival. In the absence of overdiagnosis, the dots would appear close to the solid line. The increasing distance between the observed and expected values by cohort of birth indicates that overdiagnosis has augmented over time.

Table 2 shows the overdiagnosis estimates and confidence intervals for cohorts born between 1935 and
1950. These estimates have been obtained by comparing cumulative incidences from 40 years of age to the last observed age in each cohort. Overdiagnosis estimates vary from $0.4 \%, 95 \%$ CI $(-8.8 \%, 12.2 \%)$ for the 1935 cohort to $46.6 \%, 95 \%$ CI $(22.7 \%, 85.2 \%)$ for the 1950 cohort.

Table 3 shows the results obtained after performing a sensitivity analysis for the overdiagnosis estimates. The largest change in overdiagnosis was observed when modifying mean sojourn time in the pre-clinical state $(\alpha)$. A pattern of mammography use caused a small change in overdiagnosis (less than a $5 \%$ change between the two extreme scenarios). Finally, the mammography sensitivity caused changes of around $1.5 \%$ in the overdiagnosis estimation.

\section{Discussion}

\section{Principal findings}

Breast cancer incidence in Catalonia has increased during the twentieth century with a more marked rise in cohorts born from 1940 to 1950 , who were 30 to 40 years old at the beginning of the $1980 \mathrm{~s}$. The progressive dissemination of mammography in Catalonia was significantly associated with this increase, once age and other cohort characteristics were considered. Our estimates of overdiagnosis ranged from $0.4 \%$ for women born in 1935 to $46.6 \%$ for women born in 1950 .

\section{Comparison with other studies Incidence trends}

Botha et al. studied breast cancer incidence and mortality trends in 16 European countries until the 

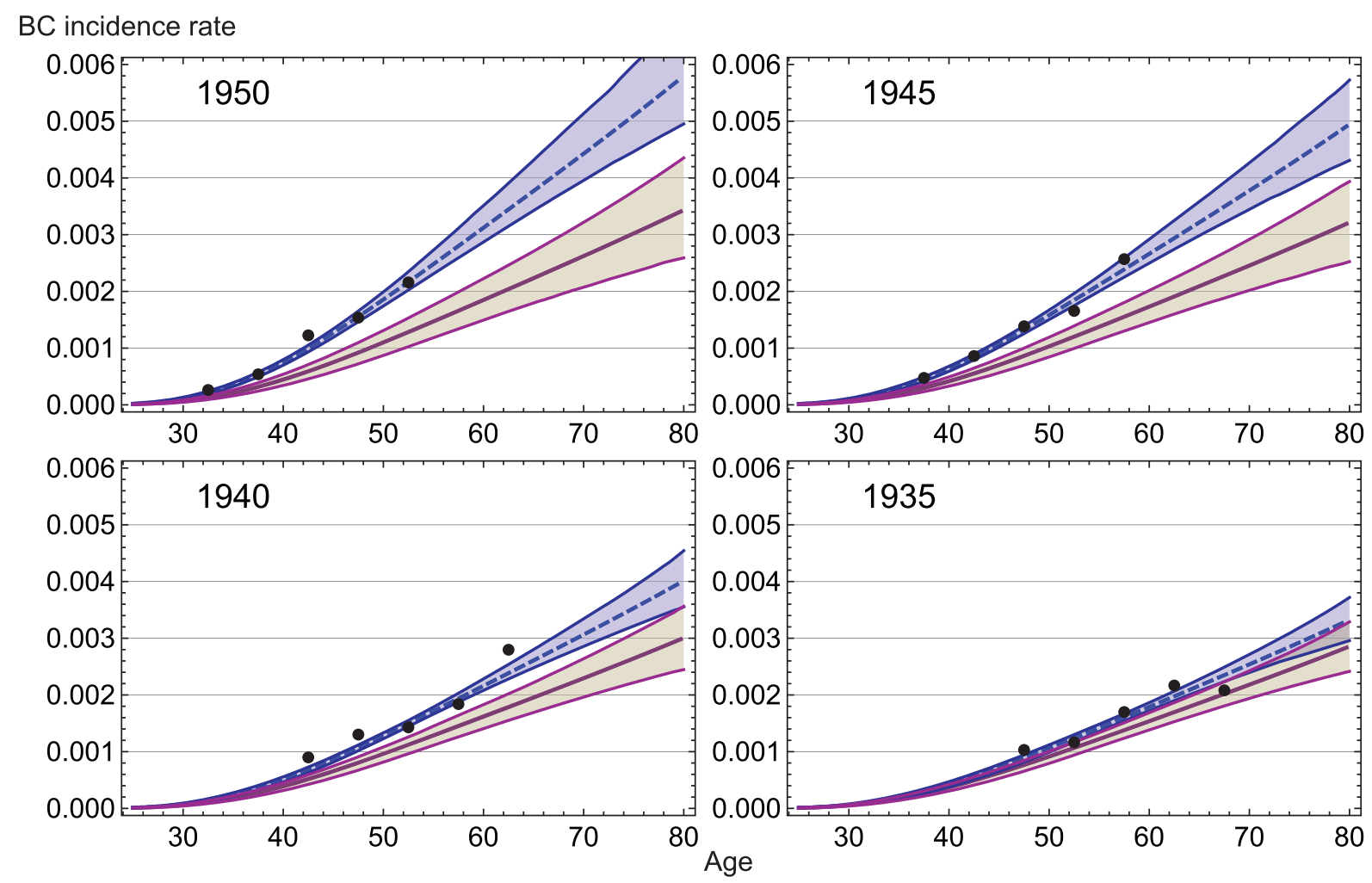

Figure 4 Breast cancer (BC) incidence model for screening and background scenarios. Each plot shows the results for cohorts born in 1935 1940, 1945 and 1950: observed BC incident rates per 100,000 women (points), model with (dashed blue line) and without (purple line) screening. Confidence intervals were obtained using bootstrap.

mid-1990 s. Increases in incidence occurred in all countries, with or without national screening programs and, according to the authors, were consistent with changes in the risk factors [6]. In the USA, Holford et al. also found an increased trend with a peak in the mid-1980 s, when screening began to be more aggressively promoted [24].

In Spain, Pollan et al. studied the trend over time of the age-adjusted incidence rate of invasive breast cancer. Incidence increased steadily during the $1980 \mathrm{~s}$ and 1990 $\mathrm{s}$ and it appeared to decline in 2000 to 2004 in the Spanish provinces were screening had achieved full coverage of the target population in a short time period [43]. In Catalonia, where dissemination of screening took longer, the change in the age adjusted incidence trend was not observed.

In contrast to the previous studies, our incidence model was not only designed to assess the trend of breast cancer incidence over time but to obtain an estimate of the background incidence and overdiagnosis. Our results show a dramatic increase of age-specific incidence rates in cohorts born after 1940 associated to the intensity of mammography use in our region.

\section{Overdiagnosis estimates}

Overdiagnosis or overdetection estimates range from negligible [15,44] or low [45-48] to moderate [22,49-51] and high (50\% or more) [14,52-55]. Some of the studies did not account for lead time bias or for decreases in incidence in older age groups no longer screened $[44,46]$.

A review of the eight randomized trials of mammography found that in recent trials in which the control group was not offered screening, an excess incidence of breast cancer remained after many years of follow-up. In those trials in which the control arm was offered screening, there was no evidence of overdiagnosis, although there was a possible shift from invasive to in situ disease [56].

Two systematic reviews intended to shed light on overdiagnosis of breast cancer. One of them, performed by Biesheuvel et al. concluded that the most reliable overdetection estimates ranged from $-4 \%$ to $21 \%$ and increased with age [22]. The other systematic review, based on published trends in incidence of breast cancer before and after the introduction of mammography screening [53], included five national screening 

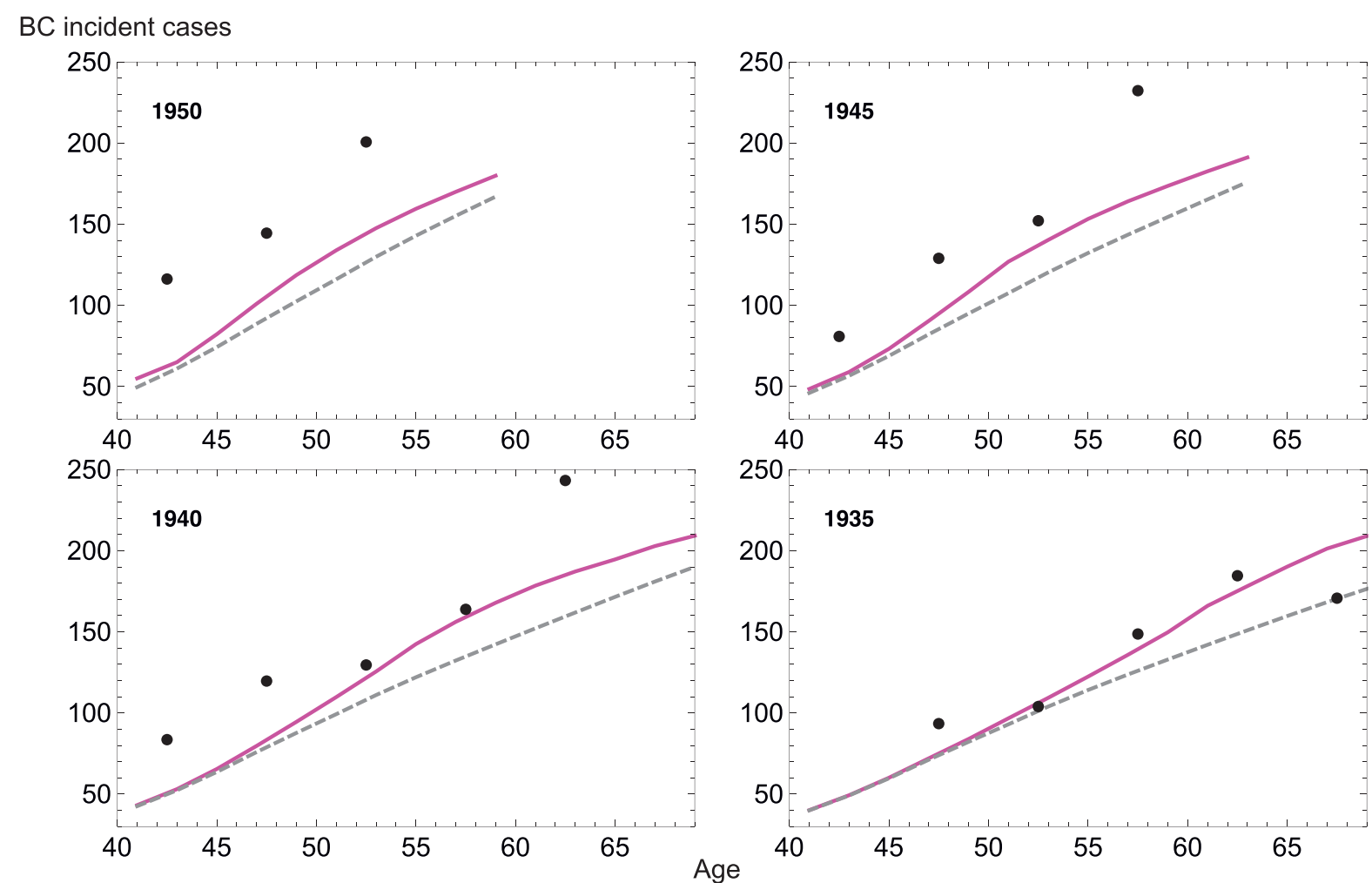

Figure 5 Predicted breast cancer incidence rates per 100,000 women at birth. Each plot shows the results for cohorts born in 1935, 1940, 1945 and 1950: observed (points), background scenario (dashed gray line), and scenario that takes into account the actual dissemination of mammography (purple line).

programs and estimated overdiagnosis in a $35 \%$ for invasive cancers.

Two recent observational studies estimated overdiagnosis using population data. Puliti et al. [57] evaluated the degree of overdiagnosis of breast cancer 15 years after the introduction of service screening in Florence (Italy). For women aged 60 to 69 years at the start of the screening, the group that had a sufficient follow-up period after the last screening, the authors did not find overdiagnosis. In contrast, Morrell et al. [58], in New South Walles (Australia) reported overdiagnosis estimates of $30 \%$ and $42 \%$ (depending on the method used) for women 50 to 69 years old. Although the study populations and methods used are different, our results are consistent with both Puliti and Morrell studies. Similarly to Puliti, we obtained no overdiagnosis for cohorts born

Table 2 Overdiagnosis estimation by year of birth in Catalonia

\begin{tabular}{lccc}
\hline Cohort & Overdiagnosis (\%) & \multicolumn{2}{c}{ [95\% conf. interval] } \\
\hline 1935 & 0.4 & -8.8 & 12.2 \\
1940 & 23.3 & 9.1 & 43.4 \\
1945 & 30.6 & 12.7 & 57.6 \\
1950 & 46.6 & 22.7 & 85.2 \\
\hline
\end{tabular}

around 1935, which were in their $60 \mathrm{~s}$ when mammography began to be widespread in Catalonia. And, consistently to Morrell, we obtained estimates of overdiagnosis higher than $40 \%$ for the younger cohorts that had been intensively exposed to mammograms for early detection.

Table 3 Sensitivity analysis for the cohort born in $\mathbf{1 9 4 5}$

\begin{tabular}{|c|c|c|}
\hline Screening pattern & Parameter change & Overdiagnosis (\%) \\
\hline Annual $(z=40)$ & - & 26.4 \\
\hline Annual $(z=40)$ & $\beta=0.9$ & 25.0 \\
\hline Annual $(z=40)$ & $\alpha=1$ & 51.1 \\
\hline Annual $(z=40)$ & $\alpha=5$ & 18.3 \\
\hline Biennial $(z=40)$ & - & 33.9 \\
\hline $\begin{array}{l}\text { Mamo dissem } 1994 \text { for } 40 \text { to } \\
49 \text { years }\end{array}$ & - & 33.8 \\
\hline $\begin{array}{l}\text { Mamo dissem } 2006 \text { for } 60 \text { to } \\
69 \text { years }\end{array}$ & - & 29.3 \\
\hline \multicolumn{3}{|c|}{$\begin{array}{l}\text { To test the sensitivity of the model we changed some of the parameters and } \\
\text { estimated overdiagnosis for woman born in } 1945 \text {. The modified parameters } \\
\text { were mammography sensitivity }(\beta) \text { and mean sojourn time in pre-clinical state } \\
(\alpha) \text {. In the first five scenarios, } 100 \% \text { of the population started receiving } \\
\text { mammography at age } 40(z=40) \text {. The last two scenarios take into account } \\
\text { the actual dissemination of mammography, and use as proportions of repeat } \\
\text { mammography behavior the most extreme vanues found in the different }\end{array}$} \\
\hline
\end{tabular}


Zahl et al., using a different approach, compared sixyear cumulative incidence of invasive breast cancer in a screened and a control group in Norway [51]. All women in the control group were invited to receive a 1time prevalence screen at the end of the observation period. Since the cumulative incidence among controls never reached that of the screened group (incidence rate ratio $=1.22$ ), the authors suggested that the natural course of some screen-detected invasive breast cancers may be to spontaneously regress. Similarly, Gotzsche et al. in the update of the Cochrane systematic review of screening for breast cancer with mammography estimated that screening led to $30 \%$ overdiagnosis and overtreatment, a figure consistent with our results [59].

\section{Strengths and weaknesses}

This study has several limitations: 1) Incidence data in Catalonia was not available at the population level. The two Catalan population based cancer registries, at the Girona and Tarragona provinces, cover an area of around $20 \%$ of the Catalan population. We have assumed that the incidence of breast cancer from these registries, was generalizable to the Catalan incidence. We think that this assumption is acceptable because even the cancer registries are geographically distant within Catalonia, the incidence estimates were close.

Besides, both cancer registries report to the International Agency for Research on Cancer and comply with its quality control procedures. 2) Incidence estimates were available for a 25 -year period, therefore some agespecific incidence rates were not available. Although the incidence model estimates were stable and precise, the estimates of overdiagnosis for some cohorts had wide confidence intervals. 3) We did not have information on trends over time of important risk factors like HRT, oral contraceptives, alcohol consumption obesity and sedentarism. We know that the use of HRT has been low in Spain. During the $1990 \mathrm{~s}$, the prevalence of HRT use among Spanish women aged 45 to 64 increased progressively reaching a value of $5.9 \%$ in 1998 and declined to $4.2 \%$ in $2006[60,61]$. Within a cohort of participants in a population-based breast cancer screening program in the city of Barcelona, the prevalence HRT peaked in 2002 at $11 \%$ in 50 - to 54 -year-olds and at $10.1 \%$ in 55 to 59-year-olds, followed by a sudden reversal and a progressive decrease [62]. Prevalence of overweight and obesity in Spain has increased as in the majority of other developed countries. A study of primary care users in the Girona province showed that the proportion of women with obesity $\left(B M I<30 \mathrm{~kg} / \mathrm{m}^{2}\right)$, in the 35 to 44 age group, increased from $6.9 \%$ in 1986 to 1989 to $12.9 \%$ in 1995 to 1999 [63]. The scarcity of information on risk factors other than fecundity and mammography use led us to include the year of birth in the model to represent the remaining cohort effect. 4) Our breast cancer incidence model used grouped data to estimate the association between incidence rates and characteristics of the exposed population at different periods of time. Grouped data analysis may be affected by the ecological fallacy or failure of aggregate level associations to properly reflect individual level associations. We intended to overcome this problem forcing the completed fertility rate variable to be inversely associated with breast cancer incidence as reported in the literature. In addition, we included the effect of year of birth in the incidence model as linear consistent with an extended increasing trend over time. This assumption provided a more conservative estimate of overdiagnosis than when we fitted a higher degree polynomial function. 5) The estimates of mean sojourn time in a preclinical state that we have used are based on data from the early detection randomized clinical trials [64] which did not take overdiagnosis into account. That would have caused mean sojourn time to appear longer than it was [54]. If mean sojourn time in a preclinical state was smaller than the values we used, our estimates of overdiagnosis would be conservative (see Table 3 where our sensitivity analysis shows the effect of changes in mean sojourn time).

The principal strength of our study is the use of probabilistic models to obtain the expected incidence of breast cancer. Based on the background incidence and the dissemination and patterns of use of mammography in Catalonia we have estimated the increased age-specific incidence due to lead time. Our study does not compare a screened group with a control group, it compares the observed incidence rates with the expected ones assuming that screening detects earlier invasive tumors that would become apparent later during the womens' life.

In comparison with the conventional age-periodcohort (APC) models, our model includes two specific cohort characteristics, the intensity of mammography use and the completed fertility rate, which have opposite trends during most of the study period. The agreement between the observed and fitted incidence rates for almost all the studied cohorts indicates the relevance of this variables when explaining incidence changes over time and the difficulties in interpreting APC models when they include only one cohort effect that summarizes divergent information.

\section{Conclusions}

Our results support the existence of overdiagnosis in breast cancer screening by mammography in Catalonia. Since our overdiagnosis estimates were high in cohorts that have not reached the age of 60 , where the impact of competing risks is low, it seems that the limited 
malignant potential of some tumors may play an important role in overdiagnosis. As other authors have recommended [20,21], women should be informed about the benefits and harms of screening and research should be oriented towards assessing individual risk and incorporating it to optimize the effectiveness of screening.

\section{Additional material}

Additional file 1: Appendix. The file contains further details of the model for dissemination of mammography, equations for the estimation of BC incidence, prevalence, mortality, and overdiagnosis.

\begin{abstract}
Abbreviations
APC: age-period-cohort; BC: breast cancer; BCSP: Catalan Breast Cancer Screening Program; CFR: completed fertility rate; CISNET: Cancer Intervention and Surveillance Modeling Network; DCIS: ductal carcinoma in situ; HRT: hormone replacement therapy; LZ: Lee and Zelen; PM50: proportion of women receiving periodic mammograms at age 50; USA: United States of America.
\end{abstract}

\section{Acknowledgements}

We thank Sandra Lee, Marvin Zelen and Hui Huang for their support in the development of the probabilistic models used. We are also grateful to the anonymous reviewers for their comments and suggestions that have improved the manuscript.

This study has been funded by grants PI06/1649, PI06/90355 and PS09/ 01340 from the Health Research Fund (Fondo de Investigación Sanitaria) of the Spanish Ministry of Health and by grant 068/27/06 of the Catalan Agency for Health Technology Assessment (Agència d'Avaluació de Tecnologia i Recerca Mèdiques).

\section{Author details}

${ }^{1}$ Basic Medical Sciences Department, Biomedical Research Institut of Lleida (IRBLLEIDA), Alcalde Rovira Roure 80, Lleida, 25198, Catalonia, Spain. ${ }^{2}$ Basic Medical Sciences Department, University of Lleida, Montserrat Roig 2, Lleida, 25008, Catalonia, Spain. ${ }^{3}$ Evaluation and Clinical Epidemiology Department, Hospital del Mar-IMIM, Doctor Aiguader 88, Barcelona, 08003, Catalonia, Spain. ${ }^{4}$ Epidemiology Unit, Girona Cancer Registry, Passatge Farinera Teixidor 1, Girona, 17005, Catalonia, Spain. ${ }^{5}$ Catalan Cancer Plan, ICO-Hospital Dr. Josep Trueta, Av. França s/n, Girona, 17007, Catalonia, Spain.

\section{Authors' contributions}

MM-A developed the age-cohort model, participated in the statistical analysis of results and interpretation, wrote drafts and obtained authors' feedback and participated in the writing of the manuscript. EV developed the computer programs that estimate the effect of screening under different scenarios, provided statistical analysis and interpretation of results and participated in the writing of the manuscript. RM-G provided the incidence data and participated in writing and revising the manuscript. MR codeveloped the project that includes this study, performed statistical analysis and participated in the writing of the manuscript.

\section{Competing interests}

The authors declare that they have no competing interests.

Received: 8 April 2010 Revised: 13 July 2010 Accepted: 3 August 2010 Published: 3 August 2010

\section{References}

1. Kelsey J, Gammon M: The epidemiology of breast cancer. CA Cancer J Clin 1991, 41:146-165.

2. Kelsey J, Gammon M, John E: Reproductive factors and breast cancer. Epidemiol Rev 1993, 15:36-47.
3. Beral V, Banks E, Reeves G, Wallis M: Hormone replacement therapy and high incidence of breast cancer between mammographic screens. Lancet 1997, 349:1103-1104

4. van den Brandt $P$, Spiegelman D, Yaun $S$, Adami $H$, Beeson L, Folsom A, Fraser G, Goldbohm R, Graham S, Kushi L, Marshall JR, Miller A, Rohan T, Smith-Warner S, Speizer F, Willett WC, Wolk A, Hunter DJ: Pooled analysis of prospective cohort studies on height, weight, and breast cancer risk. Am J Epidemiol 2000, 152:514-527.

5. Rossouw J, Anderson G, Prentice R, LaCroix A, Kooperberg C, Stefanick M, Jackson R, Beresford S, Howard B, Johnson K, Kotchen J, Ockene J, for the Women's Health Initiative Investigators WG: Risks and benefits of estrogen plus progestin in healthy postmenopausal women: principal results from the Women's Health Initiative randomized controlled trial. JAMA 2002, 288:321-333.

6. Botha J, Bray F, Sankila R, Parkin D: Breast cancer incidence and mortality trends in 16 European countries. Eur J Cancer 2003, 39:1718-1729.

7. Colditz G, Rosner B, Chen W, Holmes M, Hankinson S: Risk factors for breast cancer according to estrogen and progesterone receptor status. J Natl Cancer Inst 2004, 96:218-228.

8. Chiu C, Morrell S, Page A, Rickard M, Brassil A, Taylor R: Population-based mammography screening and breast cancer incidence in New South Wales, Australia. Cancer Causes Control 2006, 17:153-160.

9. Rosenberg L, Zhang Y, Coogan PF, Strom BL, Palmer J: A case-control study of oral contraceptive use and incident breast cancer. Am J Epidemiol 2009, 169:473-479.

10. Marshall SF, Clarke CA, Deapen D, Henderson K, Largent J, Neuhausen SL, Reynolds P, Ursin G, Horn-Ross PL, Stram DO, Templeman C, Bernstein L: Recent breast cancer incidence trends according to hormone therapy use: the California Teachers Study cohort. Breast Cancer Res 2010, 12:R4.

11. International Agency for Research on Cancer (IARC): Cancer incidence in five continents.[http://www-dep.iarc.fr].

12. Izquierdo A, Gispert R, Saladie F, Espinas J: Analysis of cancer incidence, survival and mortality according to the main tumoral localizations, 19852019: breast cancer. Med Clin (Barc) 2008, 131(Suppl 1):50-52.

13. Glass A, Lacey JJV, Carreon J, Hoover R: Breast cancer incidence, 19802006: combined roles of menopausal hormone therapy, screening mammography, and estrogen receptor status. J Natl Cancer Inst 2007, 99:1152-1161.

14. Jonsson $H$, Johansson $R$, Lenner $P$ : Increased incidence of invasive breast cancer after the introduction of service screening with mammography in Sweden. Int J Cancer 2005, 117:842-847.

15. Svendsen A, Olsen A, von Euler-Chelpin M, Lynge E: Breast cancer incidence after the introduction of mammography screening: what should be expected? Cancer 2006, 106:1883-1890.

16. Shen $Y$, Zelen M: Robust modeling in screening studies: estimation of sensitivity and preclinical sojourn time distribution. Biostatistics 2005, 6:604-614

17. Davidov O, Zelen M: Overdiagnosis in early detection programs. Biostatistics 2004, 5:603-613.

18. Duffy $S$, Lynge $E$, Jonsson $H$, Ayyaz $S$, Olsen A: Complexities in the estimation of overdiagnosis in breast cancer screening. Br J Cancer 2008, 99:1176-1178.

19. Mandelblatt J, Cronin K, Bailey S, Berry D, de Koning H, Draisma G, Huang H, Lee S, Munsell M, Plevritis S, Ravdin P, Schechter C, Sigal B, Stoto M, Stout N, van Ravesteyn N, Venier J, Zelen M, Feuer E, of the Cancer Intervention BCWG, Network SM: Effects of mammography screening under different screening schedules: model estimates of potential benefits and harms. Ann Intern Med 2009, 151:738-747.

20. Esserman $L$, Shieh $Y$, Thompson I: Rethinking screening for breast cancer and prostate cancer. JAMA 2009, 302:1685-1692.

21. Welch HG, Black WC: Overdiagnosis in cancer. J Natl Cancer Inst 2010, 102:605-613.

22. Biesheuvel C, Barratt A, Howard K, Houssami N, Irwig L: Effects of study methods and biases on estimates of invasive breast cancer overdetection with mammography screening: a systematic review. Lancet Oncol 2007, 8:1129-1138.

23. Estoesta J, Gao K, Taylor R, Ho C, Brassil A: BreastScreen New South Wales statistical report: 1999-2002. Tech rep BreastScreen NSW 2004.

24. Holford T, Cronin K, Mariotto A, Feuer E: Changing patterns in breast cancer incidence trends. J Natl Cancer Inst Monogr 2006, 36: 19-25. 
25. Berry D, Cronin K, Plevritis S, Fryback D, Clarke L, Zelen M, Mandelblatt J, Yakovlev A, Habbema J, Feuer E, Intervention C, Collaborators SMNC: Effect of screening and adjuvant therapy on mortality from breast cancer. $N$ Engl J Med 2005, 353:1784-1792.

26. Feuer E: Modeling the impact of adjuvant therapy and screening mammography on US breast cancer mortality between 1975 and 2000: introduction to the problem. J Natl Cancer Inst Monogr 2006, , 36: 2-6.

27. Lee S, Zelen M: A stochastic model for predicting the mortality of breast cancer. J Natl Cancer Inst Monogr 2006, , 36: 79-86.

28. Lee $\mathrm{S}$, Zelen M: Mortality modeling of early detection programs. Biometrics 2008, 64:386-395

29. Masuet $C$, Seculi E, Brugulat $P$, Tresserras $R$ : The practice of preventive mammography in Catalonia [Spain]: a step forward. Gac Sanit 2004, 18:321-325.

30. Marcos-Gragera R, Cardo X, Galceran J, Ribes J, Izquierdo A, Borras J: Cancer incidence in Catalonia, 1998-2002. Med Clin (Barc) 2008, 131(Suppl 1):4-10.

31. Institut d'Estadística de Catalunya: Padró municipal d'habitants. 2007 [http://www.idescat.net].

32. Cabré A: The reproduction of Catalan generations 1856-1960. PhD thesis Universitat Autònoma de Barcelona 1989.

33. Kalache A, Maguire A, Thompson S: Age at last full-term pregnancy and risk of breast cancer. Lancet 1993, 341:33-36.

34. Rue M, Carles M, Vilaprinyo E, Martinez-Alonso M, Espinas J, Pla R, Brugulat $P$ : Dissemination of periodic mammography and patterns of use, by birth cohort, in Catalonia (Spain). BMC Cancer 2008, 8:336.

35. Royston P, Altman D: Regression using fractional polynomials of continuous covariates: parsimonious parametric modelling. J R Stat Soc Ser C (Appl Stat) 1994, 43:429-467.

36. Magnusson C, Persson I, Baron J, Ekbom A, Bergstrom R, Adami H: The role of reproductive factors and use of oral contraceptives in the aetiology of breast cancer in women aged 50 to 74 years. Int J Cancer 1999, 80:231-236.

37. Rosenberg M: Competing risks to breast cancer mortality. J Natl Cancer Inst Monogr 2006, , 36: 15-19.

38. Vilaprinyo E, Gispert R, Martinez-Alonso M, Carles M, Pla R, Espinas J, Rue M: Competing risks to breast cancer mortality in Catalonia. BMC Cancer 2008, 8:331.

39. Vilaprinyo E, Rue M, Marcos-Gragera R, Martinez-Alonso M: Estimation of age- and stage-specific Catalan breast cancer survival functions using US and Catalan survival data. BMC Cancer 2009, 9:98.

40. Lee $\mathrm{S}$, Zelen M: Scheduling periodic examinations for the early detection of disease: applications to breast cancer. J Am Stat Assoc 1998, 93:1271-1281

41. StataCorp: Stata Statistical Software: Release 10. Texas 2007.

42. Wolfram Research, Inc: Mathematica Grid Edition Version 6.0.1. Illinois 2007.

43. Pollan M, Pastor-Barriuso R, Ardanaz E, Arguelles M, Martos C, Galceran J, Sanchez-Perez MJ, Chirlaque MD, Larranaga N, Martinez-Cobo R, Tobalina MC, Vidal E, Marcos-Gragera R, Mateos A, Garau I, Rojas-Martin MD, Jimenez R, Torrella-Ramos A, Perucha J, Perez-de Rada ME, Gonzalez S, Rabanaque MJ, Borras J, Navarro C, Hernandez E, Izquierdo A, LopezAbente G, Martinez C: Recent changes in breast cancer incidence in Spain, 1980-2004. J Natl Cancer Inst 2009, 101:1584-1591.

44. Olsen A, Jensen A, Njor S, Villadsen E, Schwartz W, Vejborg I, Lynge E: Breast cancer incidence after the start of mammography screening in Denmark. Br J Cancer 2003, 88:362-365.

45. de Koning H, Draisma G, Fracheboud J, de Bruijn A: Overdiagnosis and overtreatment of breast cancer: microsimulation modelling estimates based on observed screen and clinical data. Breast Cancer Res 2006, 8:202.

46. Paci $E$, Miccinesi $G$, Puliti $D$, Baldazzi $P$, De Lisi V, Falcini F, Cirilli $C$, Ferretti $S$, Mangone L, Finarelli AC, Rosso S, Segnan N, Stracci F, Traina A, Tumino R, Zorzi M: Estimate of overdiagnosis of breast cancer due to mammography after adjustment for lead time. A service screening study in Italy. Breast Cancer Res 2006, 8:R68.

47. Olsen A, Agbaje O, Myles J, Lynge E, Duffy S: Overdiagnosis, sojourn time, and sensitivity in the Copenhagen mammography screening program. Breast J 2006, 12:338-342.

48. Zackrisson S, Andersson I, Janzon L, Manjer J, Garne J: Rate of overdiagnosis of breast cancer 15 years after end of Malmo mammographic screening trial: follow-up study. BMJ 2006, 332:689-692.

49. Moller B, Weedon-Fekjaer $H$, Hakulinen $T$, Tryggvadottir $L$, Storm HH, Talback $M$, Haldorsen $T$ : The influence of mammographic screening on national trends in breast cancer incidence. Eur J Cancer Prev 2005 14:117-128.

50. Anderson W, Jatoi l, Devesa S: Assessing the impact of screening mammography: breast cancer incidence and mortality rates in Connecticut (1943-2002). Breast Cancer Res Treat 2006, 99:333-340.

51. Zahl $P$, Maehlen J, Welch $\mathrm{H}$ : The natural history of invasive breast cancers detected by screening mammography. Arch Intern Med 2008, 168:2311-6.

52. Zahl P, Strand B, Maehlen J: Incidence of breast cancer in Norway and Sweden during introduction of nationwide screening: prospective cohort study. BMJ 2004, 328:921-924.

53. Jorgensen K, Gotzsche P: Overdiagnosis in publicly organised mammography screening programmes: systematic review of incidence trends. BMJ 2009, 339:b2587.

54. Gotzsche P, Jorgensen K, Maehlen J, Zahl P: Estimation of lead time and overdiagnosis in breast cancer screening. Br J Cancer 2009, 100:219, author reply 220

55. Zahl P, Jorgensen K, Maehlen J, Gotzsche P: Biases in estimates of overdetection due to mammography screening. Lancet Oncol 2008 , 9:199-201, author reply 201-202.

56. Moss S: Overdiagnosis and overtreatment of breast cancer: overdiagnosis in randomised controlled trials of breast cancer screening. Breast Cancer Res 2005, 7:230-234

57. Puliti D, Zappa M, Miccinesi G, Falini P, Crocetti E, Paci E: An estimate of overdiagnosis 15 years after the start of mammographic screening in Florence. Eur J Cancer 2009, 45:3166-3171.

58. Morrell S, Barratt A, Irwig L, Howard K, Biesheuvel C, Armstrong B: Estimates of overdiagnosis of invasive breast cancer associated with screening mammography. Cancer Causes Control 2010, 21:275-282.

59. Gotzsche $P$, Nielsen M: Screening for breast cancer with mammography. Cochrane Database Syst Rev 2009, , 4: CD001877.

60. Benet Rodriguez M, Carvajal Garcia-Pando A, Garcia Del Pozo J, Alvarez Requejo A, Vega Alonso T: Hormonal replacement therapy in Spain. Med Clin (Barc) 2002, 119:4-8.

61. Ministerio de Sanidad y Consumo INE: Encuesta Nacional de Salud de España 2006. Tech rep 2007.

62. Barbaglia G, Macia F, Comas M, Sala M, del Mar Vernet M, Casamitjana M Castells $X$ : Trends in hormone therapy use before and after publication of the Women's Health Initiative trial: 10 years of follow-up. Menopause 2009, 16:1061-1064.

63. Fernandez-Real J, Saez M, Garcia-Rafanell J, Marques A, Serra D, Girona R, Vinets C, Andreu M, Badosa P, Faixedas D, Faixedas M, Garrido J, GomezMatai M, Torra M, Barcelo M, Saurina C, Ricart W: Ponderal evolution in the Girona population, 1989-1999. Rev Clin Esp 2003, 203:57-63.

64. Shen $Y$, Zelen M: Screening sensitivity and sojourn time from breast cancer early detection clinical trials: mammograms and physical examinations. J Clin Oncol 2001, 19:3490-3499.

doi:10.1186/bcr2620

Cite this article as: Martinez-Alonso et al:: Breast cancer incidence and overdiagnosis in Catalonia (Spain). Breast Cancer Research 2010 12:R58.

\section{Submit your next manuscript to BioMed Central and take full advantage of:}

- Convenient online submission

- Thorough peer review

- No space constraints or color figure charges

- Immediate publication on acceptance

- Inclusion in PubMed, CAS, Scopus and Google Scholar

- Research which is freely available for redistribution 